\section{Validity of the French form of the somatosensory amplification scale in a non-clinical sample}

\author{
Morgiane Bridou, Colette Aguerre \\ Department of Psychology, François \\ Rabelais University, France
}

\section{Abstract}

The SomatoSensory Amplification Scale (SSAS) is a 10-item self-report instrument designed to assess the tendency to detect somatic and visceral sensations and experience them as unusually intense, toxic and alarming. This study examines the psychometric properties of a French version of the SSAS in a nonclinical population and, more specifically, explores its construct, convergent and discriminant validities. The SSAS was completed by 375 university students, together with measures of somatization propensity (SCL-90-R somatization subscale) and trait anxiety (STAI Y form). The results of principal component and confirmatory factor analyses suggest that the French version of the SSAS evaluates essentially a single, robust factor (Somatosensory amplification) and two kinds of somatic sensitivity (Exteroceptive sensitivity and Interoceptive sensitivity). Somatosensory amplification correlated with somatization tendency and anxiety propensity. These results encourage further investigations in French of the determinants and consequences of somatosensory amplification, and its use as a therapeutic strategy.

\section{Introduction}

The concept of somatosensory amplification refers to the tendency to experience somatic and visceral sensations as unusually intense, noxious and disturbing. ${ }^{1}$ It has three main components: ${ }^{1}$ i) hypervigilance towards unpleasant bodily sensations; ii) selective focus on some relatively insignificant and rare somatic sensations; iii) a tendency to develop affects and cognitions in response to bodily changes and variations, amplifying sensations to make them more disturbing and alarming than they actually are. It involves sustained self-examination and increased attention and vigilance to unpleasant somatic sensations, a predisposition to observe the weakest and rarest of somatic sensations, and a tendency to interpret them as dangerous signs of disease without any rational argument. ${ }^{2}$ Somatosensory amplifica- tion theoretically has the properties of both a trait and a state. Indeed, although the propensity to amplify seems relatively stable over the long term (individual's characteristic baseline level), the sensitivity to bodily sensations can fluctuate within the same individual over time. ${ }^{2}$

Somatosensory amplification is especially noticeable among hypochondriacs.3,4 It has been proposed as an intermediary phenomenon that occurs after detecting benign bodily sensations and precedes hypochondriacal beliefs and thoughts.5,6 However, it may also refer to a general process of somatic symptom reporting and health appraisal. ${ }^{7}$ Nakao and Barsky suggested that the differences in somatic symptomatology among patients suffering from the same serious disease could be explained by the variability in somatosensory amplification. ${ }^{6}$ For example, reports of symptoms by patients with upper respiratory tract infections, 1,8 or chronic pain were associated with high scores on the SSAS.9,10 The concept of somatosensory amplification is also interesting because it gives a clearer understanding of why somatic symptoms are disproportionate to demonstrable organ pathology. A large number of studies have highlighted the pathogenic role of somatosensory amplification in the somatisation process. ${ }^{3,11-13}$ Currently, it also tends to be viewed as a major component of the mechanism underlying health anxiety. $14-16$

Many studies have demonstrated that somatosensory amplification is a cognitive phenomenon rather than specific somatic sensitivity. 11,17-19 Bodily information processing appears to be of particular importance. For example, Takayanagi and Fujiu identified an attentional bias toward illness-related words in individuals with high somatosensory amplification. ${ }^{20}$ Aronson, et al. ${ }^{11-12}$ suggested that this finding confirms the existence of a negatively biased reporting style (neuroticism, negative affectivity), which leads to a misappraisal of reality. Other researchers argue that somatosensory amplification is probably similar to negative emotionality and general distress, 7,21 because it is highly associated with anxiety or depression. ${ }^{6}$ Somatosensory amplification propensity is also often linked with an alexithymic tendency, a personality construct characterized by difficulties in identifying and distinguishing emotions and bodily sensations. ${ }^{22}$

By allowing the intensity of various common symptoms to be quantified, a self-report measure of somatosensory amplification can help eliminate the subjective judgment of physicians. To this end, Barsky et al. ${ }^{2}$ created the SomatoSensory Amplification Scale (SSAS). This tool can be completed quickly (less than 10 minutes) and easily. However, whether the SSAS really captures the concept of somatosensory amplification is debatable.11,12 Theoretically, it is a self-report questionnaire
Correspondence: François Rabelais University, UFR Arts \& Sciences Humaines, Department of Psychology, EA 2114 Psychologie des Ages de la Vie, 3 rue des Tanneurs, BP 4103, 37041 Tours Cedex 01, France.

Tel. +33.247366685 - Fax: +33.247366484 .

E-mail: morgiane.bridou@univ-tours.fr

Key words: french adaptation, somatosensory amplification scale, psychometric properties, validity.

Acknowledgements: we would like to thank Elizabeth Yates for her participation to this article.

Contributions: MB, has conducted the study and wrote this article; CA has coordinated the study and corrected this article.

Conflict of interests: the authors declare no potential conflict of interests.

Received for publication: 1 November 2012.

Revision received: 10 December 2012.

Accepted for publication: 11 December 2012.

This work is licensed under a Creative Commons Attribution NonCommercial 3.0 License (CC BYNC 3.0).

CC Copyright M. Bridou and C. Aguerre, 2013

Licensee PAGEPress, Italy

Health Psychology Research 2013; 1:e11

doi:10.4082/hpr.2013.e11

designed to assess the propensity to detect and focus on normal physiological states and/or uncomfortable bodily sensations and automatically interpret them as signs of a serious disease. The original version of the SSAS has good internal consistency (Cronbach's=0.82). However, according to Aronson et al.,11 it is not so much a measure of somatic sensitivity as an index of negative emotionality or general distress. Similarly, Wise and Mann considered that the SSAS focuses particularly upon a perceptual style. ${ }^{21}$ These various observations raise the question of the convergent and discriminate validity of this instrument, as well as its use to assess somatization propensity. Moreover, evidence regarding the convergent and concurrent validity of the SSAS is conflicting. ${ }^{23}$

However, the reliability and validity of several foreign adaptations of the SSAS has been validated. There are currently versions in Spanish, 17 Korean, 18 Japanese, ${ }^{24}$ Kannada language, ${ }^{25}$ Italian, ${ }^{26}$ Turkish, ${ }^{27}$ and Hungarian. ${ }^{28}$ Surprisingly, there is no validated French form of the SSAS to date, despite the interest of this instrument. The aims of this article are to present the construct validity of the French adaptation of the SSAS in a non-clinical population and to clarify exactly what it assesses. We hypothesized that somatosensory amplifi- 
cation is a good indicator of the somatization tendency (convergent validity), and that it is a construct that must be distinguished from anxiety propensity (discriminant validity).

\section{Materials and Methods}

\section{Participants and procedure}

We adapted the original American version of the SSAS for use with French speakers, ${ }^{2}$ following published recommendations. ${ }^{29} \mathrm{~A}$ forward and backward translation procedure was used. First, one bilingual investigator translated the SSAS from English into French. This preliminary version was then evaluated by two psychologists specialized in health psychology (the authors of this article) who discussed and corrected discrepancies, verified the clarity of the French items and evaluated whether relevant cultural adaptations applied needed to be made. A back translation was then carried out by a second professional translator to verify that our version matched the original, and that the meaning of the original version of the SSAS had not been changed or lost in the translation process.

Then, 375 undergraduate students participated at the François Rabelais University of Tours by connecting to an Internet site; $84.26 \%$ were following a degree course and $15.73 \%$ a master's degree. The procedure and code for accessing the website were given to the students at the start of the academic year. None of the participants were remunerated, and all were informed that their responses would remain anonymous.

\section{Measures}

The SomatoSensory Amplification Scale (SSAS) is a 10-item instrument developed by Barsky, et al. ${ }^{2}$ Respondents are asked to rate the degree to which each assertion is characteristic of you in general on an ordinal scale from 1 (Not at all true) to 5 (Extremely true). In the original version, a total amplification score is obtained by adding the scores (ranging from 10 to 50). High scores indicate a greater tendency to intensify somatic sensations.

The French somatization subscale of the Symptom Check List-90-Revised (SCL-90-R som) was used to measure somatization.30,31 Scores for seven items were added. Each self-reported item was rated on a scale of 0 (not at all distressed by the item) to 4 (extremely distressed by the item). Participants were asked to describe the intensity of symptoms experienced during the previous week. The higher the score, the greater the somatization propensity.

Trait anxiety was measured using the French adaptation of the Spielberger State-Trait Anxiety Inventory (STAI Y form), a self-report questionnaire that evaluates the propensity to develop anxiety in various stressful situations. ${ }^{32,33}$ Reliability, construct validity, and utility for distress screening have been widely documented in several French samples. ${ }^{33}$ Individuals responded to 20 questions indicating how they feel in general. Total scores range from 0 to 60 . A higher total score in the STAI indicates greater anxiety in general.

\section{Statistical analysis}

Electronic data were analyzed using Statistica 6 for Windows. Several exploratory factor analyses were used to identify the different dimensions of the SSAS. The number of factors to be extracted was established by examining the screen plot and considering eigen values greater than 1 . Items displaying an unambiguous loading of at least 0.5 on one of the factors were included on that factor. Items also had to be conceptually coherent with other items on the factor. Different dimensions of the SSAS were explored through factor analysis (principal components with varimax rotation, followed by oblique rotations).

Confirmatory factor analysis (CFA) was then performed to test the relationship between latent variables and their constituent indicators and to validate the best factor structure. Goodness-of-fit measures were designed to indicate the general overall model fit with respect to the sample data and variances. Mueller suggested that there is no single or omnibus goodness-of-fit measure to analyze data; 34 different measures need to be calculated, collectively contributing to test the model. The following analyses were therefore per-

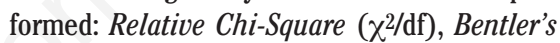
Comparative Fit Index (CFI), Standardized Root Mean Square Residual (SRMS), Root Mean Square Error of Approximation (RSMEA), Goodness of Fit Index (GFI), Adjusted Goodness of Fit Index (AGFI), Akaike's Information Criterion (AIC), and the Expected Cross-Validation Index (ECVI). Internal consistency was analyzed using Cronbach's alpha coefficients. ${ }^{35}$ Pearson correlations were calculated to analyze the relationships between somatosensory amplification and the other psychometric variables considered (somatization and anxiety) in order to test convergent and discriminant validities.

\section{Results}

\section{Descriptive analysis}

The complete research protocol was completed by 211 psychology students. The gender composition was $93.36 \%$ female and $6.64 \%$ male. Average age was 20.79 years $(\mathrm{SD}=4.19)$. The SSAS was completed by 375 psychology students. The gender composition was $65.07 \%$ female and $34.93 \%$ male. Average age was 20.79 years $(\mathrm{SD}=3.80)$. As shown in Table 1 , women reported mean scores on SSAS and STAI Y form significantly higher than men in this study, respectively $t(369)=7.44, \mathrm{P}<0.001$; $t(209)=3.49, \mathrm{P}<0.001$. On the other hand, there's no significant gender difference concerning mean scores on SCL-90-R-som, $t(209)=1.70, N S$.

\section{Construct validity}

Kaiser's measure of sampling adequacy was 0.875 . This indicated that the SSAS items were suitable for principal components analysis. ${ }^{36} \mathrm{~A}$ first factorial analysis of responses was conducted to extract a global dimension including all the SSAS items. This single factor model refers to the somatosensory amplification phenomenon and accounted for $37.3 \%$ of the total variance. In the one-factor model, all factor loadings were significant and ranged from 0.51 to 0.68 .

A second factorial analysis identified two factors, of almost equal importance, explaining $56.75 \%$ of the total variance. The first, Exteroceptive sensitivity, accounted for $28.76 \%$ of the variance and included items 1, 3, 5, 7, 8, 9 and 10. The second factor, Interoceptive sensitivity, accounted for $19.45 \%$ of the variance and included items 2,4 and 6 . In the two-factor model, as for the one-factor model, all factor loadings were significant and superior to 0.51 .

The Cronbach's alpha for the total SSAS score $(\alpha=0.81)$ indicated good reliability. It

Table 1. Descriptive results $(\mathrm{N}=375)$.

\begin{tabular}{lccc} 
& SSAS & SCL-90-R som & STAI Y form \\
Range (min-max) & $13.00-46.00$ & $0.00-24.00$ & $5.00-60.00$ \\
Mean (SD) & $30.87(7.12)$ & $7.17(4.81)$ & $27.18(9.15)$ \\
\hline Women & $32.75(7.09)$ & $7.32(4.86)$ & $27.76(8.86)$ \\
Men & $27.37(5.76)$ & $5.07(3.54)$ & $19.14(9.65)$ \\
\hline
\end{tabular}

SSAS, SomatoSensory Amplification Scale; SCL-90-R som, Symptom Check List-90-Revised somatization subscale; STAI Y form, Spielberger Trait Anxiety Inventory Y form. 
should also be noted that no item would give rise to an increase in the Cronbach's alpha if deleted. Internal consistency coefficients for Exteroceptive sensitivity $(\alpha=0.78)$ and Interoceptive sensitivity $(\alpha=0.61)$ indicated acceptable reliability, given the fact that these factors are easily interpretable. Component loadings, eigenvalues and Cronbach's alphas are presented in Table 2.

Two competing CFA models were compared. The one-factor model considers that SSAS items are represented by one global dimension called Somatosensory amplification. The two-factors model considers that SSAS items are represented by two factors: Exteroceptive sensitivity and Interoceptive sensitivity. Table 3 presents all of the fit indices for the two models.

Although all of the fit indices were close to the above-stated standards for goodness-of-fit, the two-dimensional model did not fit the data well, as all of the fit indices were well below the above-stated standards for goodness-of-fit. CFA results of the one-factor model, suggesting that this model was adequate. As shown in
Table 3, CFA results for the one-factor model show a better fit. Furthermore, comparison of the chi-squares reveals that the one-factor model was slightly better than the two-factors model, although the one-factor model accounted for a lower explained variance than the twofactors model.

\section{Convergent and discriminant validity analyses}

Pearson's correlations between the total SSAS scores and scores for the additional instruments (SCL-90-R somatization subscale and STAI Y form) are presented in Table 4. As anticipated, the somatosensory amplification tendency correlated positively with somatization propensity. However, this relationship was moderate $(\mathrm{r}=0.21, \mathrm{P}<0.05)$. We also observed a positive and significant correlation between SSAS scores and STAI scores, although this relationship was weak $(\mathrm{r}=0.19, \mathrm{P}<0.05)$. Thus, our hypothesis that somatosensory amplification is not associated with trait anxiety was not fully confirmed.

Table 2. Component loadings and eigenvalues of the 10 items on the SomatoSensory Amplification Scale (SSAS) ( $=375)$.

\begin{tabular}{|c|c|c|c|}
\hline \multirow[t]{2}{*}{ Items } & \multicolumn{3}{|c|}{ Factor loading } \\
\hline & $\begin{array}{l}\text { 1-factor model } \\
\text { loadings }\end{array}$ & $\begin{array}{r}\text { 2-fact } \\
\text { I }\end{array}$ & $\begin{array}{l}\text { model } \\
\text { igs } \\
\text { II }\end{array}$ \\
\hline 10. I can't stand pain. & -0.63 & 0.73 & \\
\hline $\begin{array}{l}\text { 9. Even something minor, like an insect bite or a splinter, } \\
\text { really bothers me. }\end{array}$ & -0.65 & 0.72 & \\
\hline 7. I hate to be too hot or too cold. & -0.66 & 0.70 & \\
\hline 8. I am quick to sense the hung contractions in my stomach. & -0.62 & 0.59 & \\
\hline 5. Sudden loud noises really bother me. & -0.68 & 0.56 & \\
\hline 3. When I bruise myself, it stays noticeable for a long time. & -0.60 & 0.54 & \\
\hline 1. I can’t stand smoke, smog or pollutants in the air. & -0.60 & 0.51 & \\
\hline 4. I sometimes can feel the blood flowing in my body. & -0.51 & & 0.81 \\
\hline $\begin{array}{l}\text { 6. I can sometimes hear my pulse or my heartbeat throbbing } \\
\text { in my ear. }\end{array}$ & -0.57 & & 0.67 \\
\hline 2. I am often aware of various things happening within my body. & -0.58 & & 0.65 \\
\hline Eigenvalues & 3.73 & 2.88 & 1.95 \\
\hline Explained variance \% & 28.76 & 19.45 & 37.3 \\
\hline
\end{tabular}

\section{Discussion}

This study aimed to investigate some of the psychometric properties of the French version of the SomatoSensory Amplification Scale (SSAS) in a non-clinical sample. Our results first support the hypothesis that the French form of the SSAS has a single dimension representing somatosensory amplification. Confirmatory factor analysis reinforced the idea that the single-factor model is better than the two-factor model, with good to excellent fit to the SSAS data. It should be noted that these findings seem to be consistent with the results of the original validation study of the American version of the SSAS which has good internal consistency (Cronbach's $\alpha=0.81$ ), ${ }^{2}$ suggesting that it could be reliably used to measure the intensification of bodily sensations in our sample. This finding is also consistent with those previously obtained by Barsky, et al. ${ }^{2}$ with medical outpatients (Cronbach's $\alpha=0.82$ ), and by Martiñez et al. ${ }^{16}$ with Spanish subjects suffering from hypochondriasis or panic attacks (Cronbach's $\alpha=0.83$ ). Speckens, Spinhoven, Sloekers, Bolk, and Van Hemert also reported an alpha coefficient of 0.71 for a non-clinical population and, ${ }^{37}$ more recently, Köteles et al.28 found internal consistency of 0.70 in a Hungarian student sample similar to ours. Based on these findings, the French version of the SSAS seems to assess the same construct as the original scale.

Our results revealed that the SSAS distinguishes between two forms of sensitivity: a tendency to perceive external stimuli acutely (Exteroceptive sensitivity), and a propensity to focus on internal information (Interoceptive sensitivity). These components are almost the same as those found earlier by Martiñez et al. 16 with a Spanish clinical population (Internal Stimulus Amplification and External Stimulus Amplification), with almost the same percentage of variance (56.75\% in our study vs $58.3 \%$ in the Spanish study). However, the factor related to interoceptive sensitivity is stronger in the Spanish study than in ours $(41.1 \%$ vs $37.3 \%$ of the variance respectively), probably because it is more representative of what people with panic or hypochondriasis disorders experience than what students feel. We there-

Table 3. Goodness of fit measures for the two compared models $(\mathrm{N}=375)$.

\begin{tabular}{|c|c|c|c|c|c|c|c|c|c|c|c|}
\hline & $x^{2}$ & df & $x^{2 / d f}$ & CFI & SRMR & RMSEA & $\begin{array}{l}\text { RMSEA } \\
90 \% \text { CI }\end{array}$ & GFI & AGFI & AIC & ECV \\
\hline 1-factor model & 86.02 & 35 & 2.46 & 0.93 & 0.048 & 0.06 & $0.05-0.09$ & 0.95 & 0.93 & 0.034 & 0.34 \\
\hline 2-factor model & 166.842 & 35 & 4.77 & 0.83 & 0.161 & 0.09 & $0.08-0.11$ & 0.93 & 0.87 & 0.56 & 0.56 \\
\hline
\end{tabular}

$\chi^{2}$, Chi-Square; df, Degrees of Freedom; CFI, Comparative Fit Index; SRMR, Standardized Root Mean Square Residual; RMSEA, Root Mean Square Error of Approximation; GFI, Goodness of Fit Index; AGFI, Adjusted Goodness of Fit Index; AIC, Akaike's Information Criterion; ECVI, Expected Cross-Validation Index. 
fore believe that identifying two kinds of bodily discomfort could be of great use in determining more precisely the supposed cause of the problem. Secondly, our results raise nosological questions, suggesting that dissimilar types of bodily sensitivities match different clinical entities. For example, we can query to what extent hypochondriasis is characterized by a particular form of somatosensory amplification, compared to the bodily sensitivity indicating a panic disorder. This should lead to more precise diagnosis (e.g. by highlighting the clinical differences between somatoform and anxiety disorders) and/or identify psychiatric comorbidities (e.g. by revealing the existence of a panic disorder characterized by interoceptive phobia). With regard to therapeutic interventions, it can be assumed that visceral awareness techniques would be more effective in decreasing excessive interoceptive sensitivity than sensitive massage techniques which would be more suitable for reducing excessive exteroceptive sensitivity.

As expected, we also found a link between total scores on the SCL-90-R somatization subscale and total scores on the SSAS. This suggests that somatosensory amplification is a reliable indicator of somatization. However, this positive correlation was moderate $(\mathrm{r}=0.21$; $\mathrm{P}<0.05)$, perhaps because our students did not experience the somatic symptoms identified by the SCL-90-R somatization subscale, but focused on lesser physical discomforts not assessed by this scale. However, our results are partially consistent with previous findings, 11 that established more robust correlations between a somatosensory amplification tendency and somatization propensity (e.g. correlations ranging from 0.41 to 0.52 in this study). This surprising finding could be explained by the low average somatization score of our French students $(7.17 ; \mathrm{SD} \pm 4.81)$ compared to those found in a similar sample (mean 17.1; $\mathrm{SD} \pm 4.7$ ) by Boujut. ${ }^{38}$ Otherwise, the discriminant validity of the SSAS is weak, in line with results of the study conducted by Aronson et $a l .11$ The fact that the SSAS is only moderately correlated with self-report measures of anxiety tendency $(\mathrm{r}=0.19 ; \quad \mathrm{P}<0.05)$ implies that somatosensory amplification and trait anxiety are not similar constructs. Both findings are consistent with the results of Wise and Mann showing a relationship between amplification and somatization, but not with anxiety. ${ }^{21}$ It should be noted here that the mean range of STAI scores was $47.18\left(\mathrm{SD}_{ \pm} 9.15\right)$, which is slightly higher than the French norms established for this sample (42.25 for men and 44.20 for women). 33

Overall, these results tend to indicate that the French form of the SSAS is: i) not really a measure of somatization as assessed by the SCL, perhaps because this tool evaluates bodily dysfunction perception and fails to identify minor somatic symptoms; and ii) a narrow indicator of psychological distress, because the STAI is a generic assessment instrument, lacking specificity. Our findings should encourage researchers to give greater importance to very small somatic disturbances that can be the focus of attention and also to consider a more specific and transient anxiety that may partially result from somatosensory amplification, namely, health anxiety. Indeed, earlier work showed that a greater tendency to worry about health tends to be associated with a greater propensity to focus on, and thereby maximize, bodily sensations. ${ }^{15}$

\section{Conclusions}

The present study suggests that our version of the SSAS is a valid and reliable measure of the propensity to focus on corporal information and attribute ordinary bodily sensations to serious somatic symptoms. A valid French instrument for measuring somatosensory amplification should encourage further work in this domain by French-speaking researchers, and more specifically, it should facilitate future research on health anxiety and somatization. It also promotes the study of the relationship between somatic and psychological symptoms.

Nonetheless, some limitations of our study must be highlighted. First, the results of this study were obtained by interviewing a cohort of university students, although Barsky et al. ${ }^{1}$

Table 4. Pearson's intercorrelations between total scores on the SSAS, the SCL-90-R somatization subscale, and Stai Y form $(\mathrm{N}=211)$.

\begin{tabular}{lccc} 
& SSAS & SCL-90-R SOm & STAI Y form \\
SSAS & 1.00 & 0.21 & 0.19 \\
SCL-90-R som & & 1.00 & 0.35 \\
\hline STAI Y form & & & 1.00 \\
\hline
\end{tabular}

All correlations are significant at $\mathrm{P}<0.05$. SSAS, SomatoSensory Amplification Scale; SCL-90-R som, Symptom Check List-90-Revised somatization subscale; STAI Y form, Spielberger Trait Anxiety Inventory Y form.

specified that the SSAS was only suitable for use with samples of medical outpatients. Nevertheless, in line with Aronson et al.,11 we believe that it could also be relevant for university students, because they generally report a number of somatic symptoms and produce high somatosensory amplification scores. In any event, these findings suggest the need for further studies to examine inter-generational differences in somatosensory amplification and somatization tendencies. More specifically, research should focus on the effects of age on somatic perception and its subjective interpretation, in order to improve our understanding of the way our relationship with our body evolves over time. It would also be interesting to investigate cross-cultural differences (e.g. between French, Spanish, Japanese, Indian, Italian, Turkish, and Hungarian samples), and to compare men and women in their awareness and interpretation of somatic information.

A second limitation of this study is that it did not evaluate the test-retest consistency of the French form of the SSAS. This is one of the main limitations of our study. More research is needed to examine the degree of temporal stability of SSAS scores and to assess to what extent the phenomenon of somatosensory amplification can be mitigated through clinical intervention to reduce health anxiety. This would help clarify whether somatosensory amplification is a stable characteristic (personality trait or perceptual style) or a phenomenon which varies according to experience (transient state), or both (as postulated by Barsky et al.). ${ }^{1}$ It may help reveal the existence of key factors of change.

Finally, this assessment tool should be tested with various clinical samples (such as people with anxiety disorders and/or somatoform disorders, hypochondriacs, people suffering from depression or chronic pain, individuals with a genetic risk of somatic disease, etc.) and with healthy samples in medical settings or exposed to potentially stressful health-related situations (e.g., medical examination, cancer screening, hospitalization, surgery, etc.). These studies would help identify particularly critical scores of somatosensory amplification, indicating great physical discomfort and psychological distress, taking into account that we do not currently have clinical cut-offs for the SSAS (as mentioned by Barsky). ${ }^{23}$ of course, we also need to be able to identify better the possible long-term deleterious consequences of somatosensory amplification (by testing the predictive validity of the SSAS), on both physical and mental health.

All these investigations will enable us to understand better the etiopathogenic factors of somatosensory amplification (life events, specific health beliefs, feedback from the environment, etc.), and their likely involvement in the processes leading to the development of 
somatization or health anxiety. In particular, greater importance should be given to cognitive modulators (dysfunctional thinking, erroneous causal attributions, cognitive errors, etc.) that may explain intrapersonal and interpersonal variations in somatosensory amplification. The cognitive-perceptual model of health anxiety could be of great heuristic value here. ${ }^{39,40}$ This postulates that individuals with significant health anxiety hold dysfunctional beliefs about health and thus misinterpret innocuous bodily sensations as dangerous. This leads to a cascade of anxiety and heightened physiological arousal which creates more symptoms and an intensification of pre-existing ones (vicious circle). Based on this explanatory model, a mindfulness-based cognitive therapy might be a promising therapeutic approach by encouraging subjects to develop their capacity to become aware of their bodily sensations with detachment (to avoid amplifying them) and also to adopt a beneficial distanced relationship with their health-related thoughts and fears. ${ }^{14}$

Ultimately, these theoretical and clinical considerations should help clinicians target their therapeutic interventions aimed at reducing somatosensory amplification more effectively, and consequently to be of greater assistance to their patients who are excessively worried about their health (hypochondriac subjects) and/or have a tendency to somatize.

\section{References}

1. Barsky AJ, Goodson JD, Lane RS, Cleary PD. The amplification of somatic symptoms. Psychosom Med 1988;50:510-9.

2. Barsky AJ, Wyshak G, Klerman GL. The somatosensory amplification scale and its relationship to hypochondriasis. J Psychiatr Res 1990;24:323-34.

3. Barsky AJ, Cleary PD, Klerman GL. Determinants of perceived health status of medical outpatients. Soc Sci Med 1992;10: 1147-54.

4. Gramling SE, Clawson EP, McDonald MK. Perceptual and cognitive abnormality model of hypochondriasis: amplification and physiological reactivity in women. Psychosom Med 1996;58:423-31.

5. Duddu V, Isaac MK, Chaturvedi SK. Somatization, somatosensory amplification, attributions styles and illness behaviour: a review. Int Rev Psychiatry 2006;18: 25-33.

6. Nakao M, Barsky AJ. Clinical application of somatosensory amplification in psychosomatic medicine. BioPsychoSocial Med 2007;1:17-23.

7. Sayar K, Barsky AJ, Güleç H. Does somatosensory amplification decrease with antidepressant treatment? Psychosomatics 2005;46:340-4.

8. Muramatsu K, Miyaoka H, Muramatsu Y, et al. The amplification of somatic symptoms in upper respiratory tract infections. Gen Hosp Psychiatry 2002;24:172-5.

9. Ak I, Sayar K, Yontem T. Alexithymia, somatosensory amplification and counterdependency in patients with chronic pain. The Pain Clinic 2004;16:43-51.

10. Kosturek A, Gregory RJ, Sousou AJ, Trief P. Alexithymia and somatic amplification in chronic pain. Psychosomatics 1998;39:399404.

11. Aronson KR, Barrett LF, Quigley KS. Feeling your body or feeling badly. Evidence for the limited validity of the somatosensory amplification scale as an index of somatic sensitivity. J Psychosom Res 2001;51:387-94.

12. Aronson KR, Barrett LF, Quigley KS. Emotional reactivity and the over report of somatic symptoms: somatic sensitivity or negative reporting style? J Psychosom Res 2006;60:521-30.

13. Fabbri S, Kapur N, Wells A, Creed F. Emotional, cognitive, and behavioural characteristics of medical outpatients: a preliminary analysis. Psychosomatics 2001;42:74-7.

14. Lovas DA, Barsky AJ. Mindfulness-based cognitive therapy for hypochondriasis, or severe health anxiety: a pilot study. J Anxiety Disord 2010;24:931-5.

15. Marcus DK, Gurley JR, Marchi MM, Bauer C. Cognitive and perceptual variables in hypochondriasis and health anxiety: a systematic review. Clin Psychol 2007;27:12739 .

16. Martiñez MP, Belloch A, Botella C. Somatosensory amplification in hypochondriasis and panic disorder. Clin Psychol Psychother 1999;6:46-53.

17. Jiménez Morón D, Saiz Ruiz J. Amplificación: somatosensorial o cognitiva? Actas Luso Esp Neurol Psiquiatr Cienc Afines 1996;24:1-6.

18. Won HT, Shin HK. A study on the cognitive characteristics of somatization (I): The reliability and validity of the Korean versions of somatosensory amplification scale and symptom interpretation questionnaire. Korean J Clin Psychol 1998;17: 33-9.

19. Nakao M, Barsky AJ, Nishikitani M, et al. Somatosensory amplification and its relationship to somatosensory, auditory, and visual evoked and event-related potentials (P300). Neurosci Lett 2007;415:185-9.

20. Takayanagi N, Fujiu H. Attentional bias toward illness threat in individuals with hypochondriacal tendency and somatosensory amplification. Jpn J Health Psychol 2008;21:12-22.
21. Wise TN, Mann LS. The relationship between somatosensory amplification, alexithymia, and neuroticism. J Psychosom Res 1994;38:515-21.

22. Nakao M, Barsky AJ, Kumano H, Kuboki T. Relationship between somatosensory amplification and alexithymia in a Japanese psychosomatic clinic. Psychosomatics 2002;43:55-60.

23. Barsky AJ. Somatosensory amplification and hypochondriasis. In: Starcevic V, Lipsitt DR, eds. Hypochondriasis: modern perspectives on an ancient malady. New York: Oxford University Press; 2001. pp 223-48.

24. Nakao M, Kumano H, Kuboki T, Barsky AJ. Reliability and validity of the Japanese version of somatosensory amplification scale: clinical application to psychosomatic illness. Jpn J Psychosom Med 2001;41:53947.

25. Duddu V, Chaturvedi SK, Isaac MK. Amplification and attribution styles in somatoform and depressive disorders. A study from Bangalore, India. Psychopathology 2003;36:98-103.

26. De Berardis D, Campanella D, Gambi F, et al. Alexithymia, fear of bodily sensations, and somatosensory amplification in young outpatients with panic disorder. Psychosomatics 2007;48:239-46.

27. Güleç H, Sayar K. Reliability and validity of the Turkish form of the somatosensory amplification scale. Psychiatry Clin Neurosci 2007;61:25-30.

28. Köteles F, Gémes H, Papp G, et al. [Validation of the Hungarian version of the Soma-tosensory Amplification Scale (SSAS)]. Mentálhigiéné es Pszichoszomatika 2009; 10:321-35. [Article in Hungarian]

29. Vallerand RJ. Vers une méthodologie de validation transculturelle de questionnaires psychologiques: implications pour la recherche en langue française. Psychologie Canadienne 1989;30:662-89.

30. Derogatis LR. Administration, scoring and procedures manual - Manual II. Towson: NCS Inc; 1983.

31. Pariente P, Guelfi JD. Inventaires d'autoévaluation de la psychopathologie chez l'adulte. lère partie: inventaires multidimensionnels. Psychiatry and Psychobiology 1990;5:49-63.

32. Spielberger CD, Gorsuch RC, Lushene RE, et al. Manual for the state-trait anxiety inventory. Palo Alto: Consulting Psychologists Press; 1983.

33. Bruchon-Schweitzer M, Paulhan I. Le manuel du STAI-Y de CD Spielberger, adaptation française. Paris: ECPA; 1993.

34. Mueller RO. Basic principles of structural equation modeling. New York: Springer Verlag; 1996. 
35. Cronbach LJ, Meehl PE. Construct validity in psychological tests. Psychol Bull 1955; 52:281-302.

36. Tabachnick BG, Fidell LS. Using multivariate statistics. New York: Harper \& Row; 1989.

37. Speckens AEM, Spinhoven P, Sloekers PPA, et al. A validation study of the whiteley index, the illness attitude scale, and the somatosensory amplification scale in gen- eral medical and general practice patients. J Psychosom Res 1996;40:95-104.

38. Boujut E. Facteurs prédisant le développement de symptômes dépressifs, de symptômes somatiques, de troubles des conduites alimentaires, de troubles des conduites alimentaires et de l'échec académique chez des étudiants de première année [Unpublished Doctoral Dissertation in Psychology]. Bordeaux
(France): University of Bordeaux II; 2007.

39. Abramowitz JS, Schwartz SA, Whiteside SP. A contemporary conceptual model of hypochondriasis. Mayo Clin Proc 2001;77: 1323-30.

40. Taylor S, Asmundson GJG. Treating health anxiety: a cognitive-behavioral approach. New York: Guildford Press; 2004. 